\title{
WHAT IS VAN DER CORPUT'S LEMMA IN HIGHER DIMENSIONS?
}

\author{
Anthony CARBERY* AND JAMES WRIGHT ${ }^{\dagger}$
}

Abstract

We consider variants of van der Corput's lemma in higher dimensions.

1. The very well-known and extremely useful van der Corput lemma is the following:

Van der Corput's lemma. Let $I \subseteq \mathbb{R}$ be an interval and suppose $\phi: I \rightarrow \mathbb{R}$ satisfies $\phi^{(k)} \geq 1$ on $I$ (where $k \in \mathbb{N}$ ). Then, for $\lambda \in \mathbb{R}$,

$$
\left|\int_{I} e^{i \lambda \phi}\right| \leq C_{k}|\lambda|^{-\frac{1}{k}},
$$

provided, in addition when $k=1$, that $\phi^{\prime}$ is monotonic on $I$.

An extensive discussion of van der Corput's lemma, its proof and its applications is given in Stein's book $[\mathbf{S}]$. Amongst the features of van der Corput's lemma emphasised in $[\mathbf{S}]$ are

- The sharpness of the decay rate (seen by taking $I=[0,1]$ and $\left.\phi(t)=t^{k} / k !\right)$.

- The fact that the constants $C_{k}$ are absolute - that is independent of $I, \lambda$ and $\phi$. This can be useful even if we do not have the sharp decay rate.

- The scaling property of the inequality: knowing the inequality for $\lambda=1$ and arbitrary $I$ automatically gives the inequality for general $\lambda$; or knowing the inequality for $I=[0,1]$ and arbitrary $\lambda$ automatically gives the inequality for general $I$. (Note that scaling can only occur because we have the sharp decay rate.)

2000 Mathematics Subject Classification. 42B99.

Key words. Oscillatory integrals, sublevel sets, multilinear forms.

* Partly supported by a Leverhulme Fellowship and by a British Council/University of Athens grant.

†Partly supported by an ARC grant. 
For the purpose of this discussion we would like to bring out two further aspects of van der Corput's lemma, as seen by examining a proof.

Proof of van der Corput's lemma: Fix a parameter $t \in(0, \infty)$ to be chosen later. Write

$$
\begin{aligned}
\int_{I} e^{i \lambda \phi} & =\int_{I \cap\left\{\left|\phi^{\prime}\right| \leq t\right\}} e^{i \lambda \phi}+\int_{I \cap\left\{\left|\phi^{\prime}\right|>t\right\}} e^{i \lambda \phi} \\
& =A+B .
\end{aligned}
$$

Now $|A| \leq\left|\left\{\left|\phi^{\prime}\right| \leq t\right\}\right|$, which, since $\left|\left(\frac{d}{d t}\right)^{k-1} \phi^{\prime}\right| \geq 1$ on $I$, can be estimated easily by induction to be dominated by $D_{k-1} t^{\frac{1}{k-1}}$, with $D_{k}$ depending only on $k$. This is a sublevel set estimate. The second term $B$ is handled by the standard integration by parts argument, working separately on each of the at most $k$ intervals upon which $\phi^{\prime}$ is monotonic, giving a contribution of $\frac{C k}{|\lambda| t}$. Optimising in $t$ gives the result.

An easy corollary of van der Corput's lemma is the sublevel set estimate (under the same hypotheses as for van der Corput's lemma)

$$
|\{s \in I|| \phi(s) \mid \leq t\}| \leq C_{k} t^{\frac{1}{k}}
$$

(with $C_{k}$ again absolute); on the other hand the proof above uses the sublevel set estimate as a principal ingredient. Thus sublevel set estimates and oscillatory integral estimates should be seen as going hand-in-hand together.

The second aspect of the proof we wish to note here is that when $k \geq 2$ there is a "hidden hypothesis". The fact that $\phi$ ' has at most $k-2$ changes of monotonicity is an immediate consequence of the hypothesis $\phi^{(k)} \geq$ $1 \geq 0$ on $I$. Thus we obtain "for free" the fact that $\phi, \phi^{\prime}, \phi^{\prime \prime}, \ldots, \phi^{(k-1)}$ all have $O(k)$ changes in monotonicity and only in the case $k=1$ do we need to explicitly require monotonicity of $\phi^{\prime}$.

In this article we wish to try to understand higher-dimensional versions of van der Corput's lemma and the associated sublevel set estimates which preserve as many as possible of the three features of the onedimensional inequality as highlighted by Stein and listed above. The first task is to formulate meaningful hypotheses. The analogue of " $\phi^{(k)} \geq 1$ " will be " $D^{\alpha} u \geq 1$ " where $u: \Omega \rightarrow \mathbb{R}$ is smooth and $\alpha$ is a multi-index. The best possible decay estimate in the sublevel set problem will be $t^{\frac{1}{|\alpha|}}$. 
(A variant of this would be to suppose that $D^{\alpha^{j}} u \geq \tau_{j}$, where $\alpha^{j}$ belongs to some prescribed set of multiindices and the $\tau_{j}$ 's are given positive numbers. See Section 4 below.) The analogue of the interval " $I$ " is the domain " $\Omega$ " of $u$, and most ambitiously we might hope to be able to let $\Omega$ be an arbitrary connected (open) set in $\mathbb{R}^{n}$. However the following example shows that some extra convexity condition is necessary. Let $n=2$ and $\alpha=(0,1)$. For $j=0,1, \ldots, N-1$ let $\Omega_{j}=\left[\frac{1}{3}, 1\right) \times\left(\frac{j}{N}, \frac{j+\frac{1}{2}}{N}\right)$, and let $\Omega=\bigcup_{j=0}^{N-1} \Omega_{j} \cup\left(0, \frac{1}{3}\right) \times(0,1)$. Let $\phi=[0,1] \rightarrow \mathbb{R}$ be smooth, $\phi \geq 0$, with $\phi \equiv 0$ on $[0,5 / 12]$ and $\phi \equiv 1$ on $[7 / 12,1]$. For $(x, y) \in \Omega_{j}$ set $u(x, y)=y-\frac{j}{N} \phi(x)$. Thus for $x \geq \frac{7}{12}, u(x, y)=y-\frac{j}{N}$ when $(x, y) \in \Omega_{j}$ and for $\frac{1}{3} \leq x \leq \frac{5}{12}, u(x, y)=y$ when $(x, y) \in \Omega_{j}$. Extend $u$ to all of $\Omega$ by setting $u(x, y)=y$ for $(x, y) \in\left(0, \frac{1}{3}\right) \times(0,1)$. Then clearly $\frac{\partial u}{\partial y} \equiv 1$ on $\Omega$ while for $t \ll \frac{1}{N},\{(x, y) \in \Omega|| u(x, y) \mid \leq t\}$ has measure like $N t$. Thus we cannot hope to work with arbitrary connected open sets and obtain absolute constants for this type of problem, and we will have to impose some conditions controlling the number of pieces that any axis parallel line may be cut into by $\Omega$. If $\Omega$ is such that any axis parallel line meets $\Omega$ in an interval, we say that $\Omega$ is $H V$-convex. Note that convex sets, in particular axis parallel rectangles, are HV-convex.

Not just the shape but also the size of the domain $\Omega$ needs to be taken into account. From the point of view of the sublevel set problem, even rectangular boxes do not constitute an adequate class of domains $\Omega$. Indeed, if we let $u(x, y)=\frac{1}{2}(x+y)^{2}$ on $(-R, R) \times(-R, R)$, then $\frac{\partial^{2} u}{\partial x d y} \equiv 1$ but

$$
|\{(x, y) \in(-R, R) \times(-R, R)|| u(x, y) \mid \leq t\}| \sim R t^{\frac{1}{2}}
$$

for large $R$. This suggests that we restrict ourselves to bounded domains $\Omega$, and that we immediately give up any ideas of scaling. In the context of oscillatory integrals, this can and has been done:

Proposition. Let $u:\left\{x \in \mathbb{R}^{n}|| x \mid<1\right\} \rightarrow \mathbb{R}$ be smooth, and suppose that $D^{\alpha} u \geq 1$ on $\{|x|<1\}$. Then $\left|\int e^{i \lambda u(x)} \phi(x) d x\right| \leq C|\lambda|^{-\frac{1}{|\alpha|}}$ for $\phi \in C_{c}^{\infty}(\{|x|<1\})$.

While the rate of decay $|\lambda|^{-\frac{1}{|\alpha|}}$ is sharp, the constant $C$ here depends on $\|u\|_{C^{|\alpha|+1}}$, as well as the test function $\phi$, and in particular this inequality exhibits no scaling properties. See $[\mathbf{S}]$.

However, the same example, $u(x, y)=\frac{1}{2}(x+y)^{2}$, suggests a remedy for this difficulty. In the context of the oscillatory integral problem, $e^{i u(x, y)}$ is essentially the kernel for the Fourier transform operator on $\mathbb{R}$. 
So we have the inequalities

$$
\left|\int e^{i \lambda u(x, y)} f(x) g(y) d x d y\right| \leq \frac{C\|f\|_{p}\|g\|_{p}}{|\lambda|^{\frac{1}{p^{\prime}}}}
$$

for $1 \leq p \leq 2$, by Plancherel's theorem and the Hausdorff-Young inequality. These inequalities are not restricted to bounded subsets of $\mathbb{R}^{2}$ and indeed scale. Admittedly the phase function is very special here, but this line of thought does suggest that one should consider the multilinear operators (in fact $n$-linear operators if we are in $\mathbb{R}^{n}$ ) whose kernels are $\chi_{\{|u| \leq t\}}$ and $e^{i \lambda u}$ respectively. In the case $n=2$ this points to work of Phong and Stein [PS2] (and the references therein) and Christ and the present authors $[\mathbf{C C W}]$.

In higher dimensions, Phong, Stein and Sturm [PSS] have recently considered the more general multilinear setting. Thus, for a suitable class of scale invariant domains $\Omega$ in $\mathbb{R}^{n}$ and for smooth $u: \Omega \rightarrow \mathbb{R}$ satisfying $D^{\alpha} u \geq 1$ on $\Omega$ we are led to the following problems:

\section{Multilinear sublevel set problem.}

Determine the values of $\gamma$ and $p_{j}$ such that

$$
\left|\int_{\Omega \cap\{|u| \leq t\}} f_{1}\left(x_{1}\right) \ldots f_{n}\left(x_{n}\right) d x_{1} \ldots d x_{n}\right| \leq C t^{\gamma} \prod_{i=1}^{n}\left\|f_{i}\right\|_{p_{i}} .
$$

\section{Multilinear van der Corput problem.}

Determine the values of $\gamma$ and $p_{j}$ such that

$$
\left|\int_{\Omega} e^{i \lambda u(x)} f_{1}\left(x_{1}\right) \ldots f_{n}\left(x_{n}\right) d x_{1} \ldots d x_{n}\right| \leq C|\lambda|^{-\gamma} \prod_{i=1}^{n}\left\|f_{i}\right\|_{p_{i}} .
$$

To gain some insight into these problems, let us fix $\Omega=Q=[0,1]^{n}$ for now. Consideration of $u(x)=\left(x_{1}+\cdots+x_{n}\right)^{|\alpha|}$ and $f_{j} \equiv 1$ shows that $\gamma$ must be less than or equal to $\frac{1}{|\alpha|}$ irrespective of the $p_{j}$ 's. Consideration of $u(x)=x_{1}^{\alpha_{1}} \ldots x_{n}^{a_{n}},{ }^{1} f_{1}=\chi_{\left(0, t^{\frac{1}{\alpha_{1}}}\right)}, f_{j} \equiv 1(j=2, \ldots, n)$ shows that $\gamma$ can be at most $\frac{1}{\alpha_{1} p_{1}^{\prime}}$. By symmetry, we therefore must have $\gamma \leq \min \left\{\frac{1}{|\alpha|}, \frac{1}{\alpha_{j} p_{j}^{\prime}}\right\}$. So when $\frac{1}{p_{j}}=1-\frac{\alpha_{j}}{|\alpha|}$ we can hope to take $\gamma=$

\footnotetext{
${ }^{1}$ It is also easy to establish bounds for the multilinear operators in these cases. For example, if $u(x)=x_{1}^{\alpha_{1}} \ldots x_{n}^{\alpha_{n}}$, then (1) holds along the line $\frac{1}{\alpha_{1} p_{1}^{\prime}}=\frac{1}{\alpha_{2} p_{2}^{\prime}}=\cdots=$ $\frac{1}{\alpha_{n} p_{n}^{\prime}}$, (except possibly for an endpoint), with $\gamma$ equal to the common value of $\frac{1}{\alpha_{j} p_{j}^{\prime}}$.
} 
$\frac{1}{|\alpha|}$; moreover from this special case all other possible inequalities would follow by interpolation with trivial estimates.

However, of these possible inequalities, only certain scale properly and are thus of current interest to us. Indeed, if for example we know that whenever $D^{\alpha} u \geq 1$ on an arbitrary (isotropic) dilate $R Q$ of the unit cube $Q$ we have

$$
\left|\int_{\{|u| \leq 1\} \cap R Q} \prod_{i=1}^{n} f_{i}\left(x_{i}\right) d x_{i}\right| \leq C \prod_{i=1}^{n}\left\|f_{i}\right\|_{p_{i}},
$$

changing variables gives, for arbitrary positive $t$,

$$
\left|\int_{\{|u| \leq t\} \cap Q} \prod_{i=1}^{n} f_{i}\left(x_{i}\right) d x_{i}\right| \leq C t^{\frac{1}{|\alpha|}\left(\frac{1}{p_{1}^{\prime}}+\cdots+\frac{1}{p_{n}^{\prime}}\right)} \prod_{i=1}^{n}\left\|f_{i}\right\|_{p_{i}} .
$$

Hence a necessary condition for scaling to occur is

$$
\frac{1}{|\alpha|}\left(\frac{1}{p_{1}^{\prime}}+\cdots+\frac{1}{p_{n}^{\prime}}\right) \leq \min \left\{\frac{1}{|\alpha|}, \frac{1}{\alpha_{j} p_{j}^{\prime}}\right\} .
$$

Thus $\sum_{j=1}^{n} \frac{1}{p_{j}^{\prime}} \leq 1$ and, setting $\theta_{j}=\frac{|\alpha|}{p_{j}^{\prime} \alpha_{j}}$ also $\sum_{j=1}^{n} \theta_{j} \alpha_{j} \leq \min \left\{\theta_{j}\left(\alpha_{1}+\right.\right.$ $\left.\left.\cdots+\alpha_{n}\right)\right\}$, which can only happen if all the $\theta_{j}$ 's are equal i.e. if $\frac{1}{\alpha_{1} p_{1}^{\prime}}=$ $\frac{1}{\alpha_{2} p_{2}^{\prime}}=\cdots=\frac{1}{\alpha_{n} p_{n}^{\prime}}$. Thus scaling can occur precisely for $\left(\frac{1}{p_{1}}, \ldots, \frac{1}{p_{n}}\right)$ lying on the line segment joining $\left(1-\frac{\alpha_{1}}{|\alpha|}, \ldots, 1-\frac{\alpha_{n}}{|\alpha|}\right)$ to $(1,1, \ldots, 1)$ with the correspondingly interpolated value of $\gamma$ being the common value of $\frac{1}{\alpha_{j} p_{j}^{\prime}}$. So it is the possible validity of estimates (1) and (2) in these circumstances which interests us.

2. We now present some results in the higher-dimensional context which preserve at least one of Stein's features.

Theorem 1. Let $Q=[0,1]^{n}$ and let $\alpha$ be a multiindex in $\mathbb{Z}_{+}^{n}$. Let $p_{1}, \ldots, p_{n}>1$. Then there exists $a C>0$ and an $\varepsilon>0$ depending only on $\alpha, n$ and $p_{1}, \ldots, p_{n}$ such that if $D^{\alpha} u \geq 1$ on $Q$, then

$$
\left|\int_{\{|u|<t\}} \prod_{i=1}^{n} f_{i}\left(x_{i}\right) d x_{i}\right| \leq C t^{\varepsilon} \prod_{i=1}^{n}\left\|f_{i}\right\|_{p_{i}} .
$$


Theorem 1 was not actually stated in $[\mathbf{C C W}]$, which concerned itself with bilinear operators, but the proof is the same as that of Theorem 3.16 of $[\mathbf{C C W}$. In general we do not have the sharp $\varepsilon$ and so there is no scaling in Theorem 1. In some cases, however, we do get the correct scale invariant result:

Theorem 2. Let $n=2$, and let $\Omega$ be an $H V$-convex domain in $\mathbb{R}^{2}$. Let $\alpha$ be a multiindex in $\mathbb{Z}_{+}^{2}$ and suppose $D^{\alpha} u \geq 1$ on $\Omega$.

(a) If $\alpha=(1,1)$ and $1 \leq p<2$ there exists an absolute constant $C_{p}$ depending only on $p$ such that

$$
\left|\int_{\{|u| \leq t\}} f_{1}\left(x_{1}\right) f_{2}\left(x_{2}\right) d x_{1} d x_{2}\right| \leq C_{p} t^{\frac{1}{p^{\prime}}}\left\|f_{1}\right\|_{p}\left\|f_{2}\right\|_{p} .
$$

(b) If $\alpha=(1,1)$, there exists a constant $C$ depending only on $\Omega$ such that

$$
\left|\int_{\{|u| \leq t\}} f_{1}\left(x_{1}\right) f_{2}\left(x_{2}\right) d x_{1} d x_{2}\right| \leq C t^{\frac{1}{2}}\left(\log \frac{1}{t}\right)^{\frac{1}{2}}\left\|f_{1}\right\|_{2}\left\|f_{2}\right\|_{2} .
$$

(c) If $\alpha=(j, k), k \geq j>1, \frac{1}{p_{1}}=\frac{k}{k+1}, \frac{1}{p_{2}}=\frac{j(k+1)-k}{j(k+1)}$, there exists an absolute constant $C$ depending only on $\alpha$ such that

$$
\left|\int_{\{|u| \leq t\}} f_{1}\left(x_{1}\right) f_{2}\left(x_{2}\right) d x_{1} d x_{2}\right| \leq C t^{\frac{1}{j(k+1)}}\left\|f_{1}\right\|_{p_{1}}\left\|f_{2}\right\|_{p_{2}} .
$$

(d) If $\alpha=(1, k), \frac{1}{p_{1}}=\frac{k}{k+1}, \frac{1}{p_{2}}=\frac{1}{k+1}$ and $\left(\frac{1}{q_{1}}, \frac{1}{q_{2}}\right)$ is on the line segment joining $\left(\frac{1}{p_{1}}, \frac{1}{p_{2}}\right)$ to $(1,1)$, there exists an absolute constant $C$ depending only on $k, q_{1}$ and $q_{2}$ such that if $\left(q_{1}, q_{2}\right) \neq\left(p_{1}, p_{2}\right)$

$$
\left|\int_{\{|u| \leq t\}} f_{1}\left(x_{1}\right) f_{2}\left(x_{2}\right) d x_{1} d x_{2}\right| \leq C t^{\frac{1}{q_{1}^{\prime}}}\left\|f_{1}\right\|_{q_{1}}\left\|f_{2}\right\|_{q_{2}}
$$

and

$\left|\int f_{1}\left(x_{1}\right) f_{2}\left(x_{2}\right) d x_{1} d x_{2}\right| \leq C_{k} t^{\frac{1}{k+1}}\left(\log \frac{1}{t}\right)^{\frac{k}{k+1}}\left\|f_{1}\right\|_{p_{1}}\left\|f_{2}\right\|_{p_{2}}$

where $C_{k}$ depends only on $k$ and $\Omega$. 
Parts (a), (c) and (d) $\left(\left(q_{1}, q_{2}\right) \neq\left(p_{1}, p_{2}\right)\right)$ are sharp scale invariant estimates. Part (b) measures what we are currently know about the endpoint $p_{1}=p_{2}=2$ in (a), and was explicitly proved in $[\mathbf{C C W}]$ where $\Omega=[0,1]^{2}$. Parts (c) and (d) $\left(\left(q_{1}, q_{2}\right)=\left(p_{1}, p_{2}\right)\right)$ were also proved in $[\mathbf{C C W}]$ in the setting $Q=[0,1]^{2}$ but the proof there extends to the general HV-convex case. Parts (a) and (d) $\left(\left(q_{1}, q_{2}\right) \neq\left(p_{1}, p_{2}\right)\right)$ have not perhaps been noted explicitly before, so we include a proof of part (a). The proof of part (d) will be similar, following the lines of Theorem 3.9 in $[\mathbf{C C W}]$.

Proof of Theorem 2(a): We write $(x, y)$ for $\left(x_{1}, x_{2}\right)$ and $(f, g)$ for $\left(f_{1}, f_{2}\right)$. We may assume $p>1$. Let $E=\{(x, y) \in \Omega|| u(x, y) \mid \leq t\}$ and $E(y)=$ $\{x \in \mathbb{R} \mid(x, y) \in E\}$ be the slice of $E$ at height $y \in \mathbb{R}$. As in [CCW], $\frac{\partial^{2} u}{\partial x \partial y} \geq 1$ on $\Omega$ implies that if $(x, y),\left(x^{\prime}, y\right),\left(x, y^{\prime}\right),\left(x^{\prime}, y^{\prime}\right)$ are all in $E$, then $\left|x-x^{\prime}\right|\left|y-y^{\prime}\right| \leq 4 t$. In particular, $\left|E(y) \cap E\left(y^{\prime}\right)\right| \leq \frac{4 t}{\left|y-y^{\prime}\right|}$. Then

$$
\begin{array}{rl}
\mid \iint_{E} & f(x) g(y) d x d y \mid \\
\leq & \left\|\int \chi_{E}(x, y) g(y) d y\right\|_{p^{\prime}}\|f\|_{p} \\
& =\left\|\left(\int \chi_{E}(x, y) g(y) d y\right)^{2}\right\|_{p^{\prime} / 2}^{\frac{1}{2}}\|f\|_{p} \\
& =\left\|\iint \chi_{E}(x, y) \chi_{E}\left(x, y^{\prime}\right) g(y) g\left(y^{\prime}\right) d y d y^{\prime}\right\|_{p^{\prime} / 2}^{\frac{1}{2}}\|f\|_{p} \\
& \leq\left(\iint\left|g(y) g\left(y^{\prime}\right)\right|\left\|\chi_{E}(x, y) \chi_{E}\left(x, y^{\prime}\right)\right\|_{L^{p^{\prime}} / 2}(d x)\right. \\
= & \left(\iint \mid g d y^{\prime}\right)^{\frac{1}{2}}\|f\|_{p} \\
\leq & \left(\iint\left|g(y) g\left(y^{\prime}\right) \| E(y) \cap E\left(y^{\prime}\right)\right|^{\frac{2}{p^{\prime}}} d y d y^{\prime}\right)^{\frac{1}{2}}\|f\|_{p} \\
& =4 t y^{\frac{1}{p^{\prime}}} t^{\frac{1}{p^{\prime}}}\|f\|_{p}\left\langle I_{\gamma} g, g\right\rangle^{\frac{1}{2}} \quad\left(\text { with } 1-\gamma=\frac{2}{p^{\prime}}\right) \\
\leq & C_{p} t^{\frac{1}{p^{\prime}}}\|f\|_{p}\|g\|_{p},
\end{array}
$$

by the Hardy-Littlewood-Sobolev theorem of fractional integration. 
The reader will notice the similarity between this proof and the Fefferman-Stein argument $[\mathbf{F}]$ for the sharp restriction estimate for the Fourier transform in two dimensions. Even in the case $f_{1}=f_{2}=1$ in part (b) of Theorem 2 on $[0,1]^{2}$ we do not currently know whether the logarithmic term is present. This is related to the following combinatorial problem, a positive solution of which would also resolve this case $f_{1}=f_{2}=1$ :

Problem. Does there exist a $C>0$ such that if $E$ is a nonempty measurable subset of $[0,1]^{2}$, then there exist 4 points in $E$ arranged as the vertices of an axis parallel rectangle $R$ with area $(R) \geq C\{\operatorname{area}(E)\}^{2}$ ?

We now turn to the oscillatory integral version of Theorem 1.

Theorem 3. Let $Q=[0,1]^{n}$ and let $\alpha$ be a multiindex in $\mathbb{Z}_{+}^{n}$ with at least two nonzero components and at least one entry with value at least 2. Let $p_{1}, \ldots, p_{n}>1$. Then there exists $a C>0$ and an $\varepsilon>0$ depending only on $\alpha, n$ and $p_{1}, \ldots, p_{n}$ such that if $D^{\alpha} u \geq 1$ on $Q$, then

$$
\left|\int_{Q} e^{i \lambda u(x)} \prod_{i=1}^{n} f_{i}\left(x_{i}\right) d x_{i}\right| \leq C|\lambda|^{-\varepsilon} \prod_{i=1}^{n}\left\|f_{i}\right\|_{p_{i}} .
$$

Once again, Theorem 3 was not actually stated in $[\mathbf{C C W}]$, but the argument is the same at that of Theorem 4.13 of $[\mathbf{C C W}]$. In no case do we get the sharp scale invariant result, although when $n=2, \alpha=(1, k)$, $k \geq 2$ and $p_{1}=p_{2}=2$ we do obtain the best value $\varepsilon=\frac{1}{2 k}$. See Theorem 4.8 of $[\mathbf{C C W}]$. The requirement that $\alpha$ have at least one entry at least 2 is needed for the same reason that the classical van der Corput lemma fails in the case $k=1$ without the supplementary monotonicity hypothesis. See $[\mathbf{C C W}]$. The requirement that $\alpha$ have at least two nonzero entries is clear as if $\alpha$ were $(2,0)$, say, we could incorporate $e^{i \lambda x_{1}^{2}}$ into $f_{1}$. (In the special case that $f_{i} \equiv 1$ this requirement is not needed.)

3. Up till now we have been trying to establish analogues of van der Corput estimates and sublevel set estimates under the sole hypothesis $D^{\alpha} u \geq 1$ on $u$. But how natural is this? After all, even in the one-dimensional case one genuinely needs to impose monotonicity of $\phi^{\prime}$ in the case $k=1$ of van der Corput's lemma (but not the sublevel set estimate) because the mere fact that $\phi^{\prime} \geq 1$ does not imply that the sublevel sets of $\phi^{\prime}$ possess any regular structure. As we saw above, the same phenomenon persists in the higher dimensional multilinear oscillatory integral scenario. In a similar vein, assuming only that $\frac{\partial^{2} u}{\partial x \partial y} \geq 1$ on 
$[0,1]^{2}$ does not seem enough to conclude that the sublevel sets of $u$ have any "manageable structure" and they may in principle be "infinitely wiggly". Added to this is the fact that under the hypothesis $\phi^{(k)} \geq 1$ on $I(k \geq 2)$ we automatically have very good control of monotonicity properties of $\phi^{\prime}, \phi^{\prime \prime}, \ldots, \phi^{(k-1)}$ for free. Thus it seems to make sense to augment the basic condition $D^{\alpha} u \geq 1$ on $\Omega$ with some "qualitative regularity conditions" on sublevel sets of $D^{\beta} u$, for certain $|\beta| \leq|\alpha|$. As a first result in this direction we have, from $[\mathbf{C C W}]$ :

Theorem 4. Let $n=2$, and let $\alpha \in \mathbb{Z}_{+}^{2}$ be a multiindex. Suppose $D^{\alpha} u \geq 1$ on $[0,1]^{2}$ and that each vertical line is cut into at most $N$ pieces by any sublevel set $\{|u|<s\}$ of $u$. Then

$$
\left|\int_{\{|u| \leq t\}} f_{1}\left(x_{1}\right) f_{2}\left(x_{2}\right) d x_{1} d x_{2}\right| \leq C t^{\frac{1}{|\alpha|}}\left\|f_{1}\right\|_{p_{1}}\left\|f_{2}\right\|_{p_{2}}
$$

where $C$ depends on $\alpha$ and $N$, and $\frac{1}{p_{i}}=1-\frac{\alpha_{i}}{|\alpha|}$.

The proof of this result is fairly complicated and uses Carleson measures, see Theorem 3.13 of $[\mathbf{C C W}]$. Although the theorem was stated and proved for the unit square $[0,1]^{2}$, it seems likely that it remains valid for any $\mathrm{HV}$-convex domain $\Omega \subseteq \mathbb{R}^{2}$.

A class of functions exhibiting "well-controlled wigglyness" is the class of polynomials of fixed degree. Phong, Stein and Sturm [PSS] have recently studied multilinear operators associated to polynomials and proved in particular the following result:

Theorem $([\mathbf{P S S}])$. Let $Q=[0,1]^{n}$ and let $u: Q \rightarrow \mathbb{R}$ be a polynomial of degree at most $d$. Then

$$
\left|\int_{Q \cap\{|u| \leq t\} \cap\left\{\left|D^{\alpha} u\right| \geq 1\right\}} \prod_{i=1}^{n} f_{i}\left(x_{i}\right) d x_{i}\right| \leq C_{n, d, \alpha} t^{\frac{1}{|\alpha|}} \log ^{n-2}\left(\frac{1}{t}\right) \prod_{i=1}^{n}\left\|f_{i}\right\|_{p_{i}}
$$

where $\frac{1}{p_{i}}=1-\frac{\alpha_{i}}{|\alpha|}$.

Even when $n=2$, Phong, Stein and Sturm use considerations related to algebraic geometry (Bézout's theorem) rather than Carleson measures. This allows them to reduce the case $n=2$ of their theorem to the corresponding result on an HV domain $\Omega \subseteq Q \subseteq \mathbb{R}^{2}$ upon which both $|u| \leq t$ and $\left|D^{\alpha} u\right| \geq 1$. They then notice that for any such domain $\Omega$ containing $0, \Omega$ is forced to be contained in $\left\{\left|x_{1}\right|^{\alpha_{1}}\left|x_{2}\right|^{\alpha_{2}} \leq t\right\}$. (This step does not require the polynomial character of $u$.) Comparison with the 
known result for $u\left(x_{1}, x_{2}\right)=x_{1}^{\alpha_{1}} x_{2}^{\alpha_{2}}$ on $[0,1]^{2}$ finishes this case $n=2$, and the higher dimensional case proceeds by an inductive argument.

In view of the preceding discussion, and in view of the example given above to explain why connectedness of $\Omega$ is not the correct notion in higher dimensions, the following definition seems natural:

Definition. Let $\Omega \subseteq \mathbb{R}^{n}$ be open, and $u: \Omega \rightarrow \mathbb{R}$ be smooth. Then $u$ is of type $M$ if for any multiindex $\gamma$ with $|\gamma| \leq M$, any axis parallel line is cut into at most $N$ pieces by any sublevel set $\left\{x \in \Omega|| D^{\gamma} u \mid \leq s\right\}$, $s \in \mathbb{R}$. (We call the least such $N$ the "type $M$ constant of $u$ ", denoted $t_{M}(u)$.)

Note that if $u: \mathbb{R}^{n} \rightarrow \mathbb{R}$ is a polynomial of degree at most $d$, then $t_{M}(u)$ is finite for each $M$ and depends on $M, n$ and $d$ but not upon the coefficients of $u$. Note also that if $u$ is of type $M$ then it is also of type $M^{\prime}$ for any $M^{\prime} \leq M$.

Theorem 5. Suppose $u: \Omega \rightarrow \mathbb{R}$ is of type $M$ and let $\alpha$ be a multiindex with $|\alpha| \leq M$. Let $\frac{1}{p_{i}}=1-\frac{\alpha_{i}}{|\alpha|}$. Then

$$
\left|\int_{\Omega \cap\{|u| \leq t\} \cap\left\{\left|D^{\alpha} u\right| \geq 1\right\}} \prod_{i=1}^{n} f_{i}\left(x_{i}\right) d x_{i}\right| \leq C t^{\frac{1}{|\alpha|}} \prod_{i=1}^{n}\left\|f_{i}\right\|_{p_{i}, 1}
$$

and

$$
\left|\int_{\Omega \cap\{|u| \leq t\} \cap\left\{\left|D^{\alpha} u\right| \geq 1\right\}} \prod_{i=1}^{n} f_{i}\left(x_{i}\right) d x_{i}\right| \leq C t^{\frac{1}{|\alpha|}} \log \left(\frac{1}{t}\right) \prod_{i=1}^{m}\left\|f_{i}\right\|_{p_{i}},
$$

where $C$ depends upon $n, \alpha, M, t_{M}(u)$, and, in case (b), also the diameter of $\Omega$.

Part (a) of Theorem 5 provides the correct optimal scale invariant estimate for type $M$ domains, with the caveat that the usual $L^{p}$ spaces are replaced by the Lorentz spaces $L^{p, 1}$. If one insists upon the usual $L^{p}$ spaces, then one obtains the desired result at the expense of a single power of $\log \left(\frac{1}{t}\right)$. An investigation of whether this logarithmic term can be removed is being undertaken by Gibson, $[\mathbf{G}]$. Part (b) is proved using a many point multilinear interpolation technique similar to the one in [CHS]. Part (a) of Theorem 5 was anticipated in a bilinear setting in $[\mathbf{C C W}]$. See the remark at the end of Section 3 of $[\mathbf{C C W}]$. 
Sketch of Proof of Theorem 5(a): The proof is by induction on $|\alpha|$ and is essentially a reworking of the one-dimensional sublevel set argument.

Case $\alpha=(1,0, \ldots, 0)$ : This is just the case $k=1, n=1$ of the sublevel set estimate when unravelled and follows from the mean-value theorem.

Note that symmetry in the hypothesis now gives the result for any $\alpha=(0,0, \ldots, 0,1,0, \ldots, 0)$.

Case $\alpha+$ Case $\beta \Rightarrow$ Case $\alpha+\beta$ : Note that

$$
\begin{aligned}
& \{|u| \leq t\} \cap\left\{\left|D^{\alpha+\beta} u\right| \geq 1\right\} \\
& \quad \subseteq\left\{|u| \leq t,\left|D^{\alpha} u\right| \geq s\right\} \cup\left\{\left|D^{\alpha} u\right| \leq s,\left|D^{\alpha+\beta} u\right| \geq 1\right\} .
\end{aligned}
$$

With $f_{i}=\chi_{E_{i}}$, then,

$$
\begin{aligned}
\int_{\Omega \cap\{|u| \leq t\} \cap\left\{\left|D^{\alpha+\beta} u\right| \geq 1\right\}} & \prod_{i} \chi_{E_{i}}\left(x_{i}\right) d x_{i} \\
\leq & \int_{\Omega \cap\{|u| \leq t\} \cap\left\{\left|D^{\alpha} u\right| \geq s\right\}} \prod_{i} \chi_{E_{i}}\left(x_{i}\right) d x_{i} \\
& +\int_{\Omega \cap\left\{\left|D^{\alpha} u\right| \leq s\right\} \cap\left\{\left|D^{\alpha+\beta} u\right| \geq 1\right\}} \prod_{i} \chi_{E_{i}}\left(x_{i}\right) d x_{i} \\
\leq & C\left(\frac{t}{s}\right)^{\frac{1}{|\alpha|}} \prod_{i}\left|E_{i}\right|^{1-\frac{\alpha_{i}}{|\alpha|}}+C s^{\frac{1}{|\beta|}} \prod_{i}\left|E_{i}\right|^{1-\frac{\beta_{i}}{|\beta|}} \\
\leq & C t^{\frac{1}{|\alpha+\beta|}} \prod_{i}\left|E_{i}\right|^{1-\frac{\alpha_{i}+\beta_{i}}{|\alpha+\beta|}},
\end{aligned}
$$

by the $\alpha$-result applied to $u$ and the $\beta$-result applied to $D^{\alpha} u$, and optimising in $s$.

Full details of Theorem 5 will appear in $[\mathbf{C W}]$.

We now examine whether a similar argument can be made to work for multilinear oscillatory integral operators. As in the proof of van der Corput's lemma, and as in Theorem 5's proof, the multilinear restrictedtype sublevel set estimate for index $\alpha$ plus the multilinear restricted-type oscillatory integral estimate for index $\beta$ give the multilinear restrictedtype oscillatory integral estimate for index $\alpha+\beta$. As the sublevel set estimates are under control we only need to start the induction with a suitable oscillatory integral estimate. The index $(1,0, \ldots, 0)$ does not 
work for oscillatory integrals (as pointed out in Section 2 above) and so the place to begin is $(1,1,0, \ldots, 0)$, or, what is effectively the same thing, $(1,1)$ in the two-dimensional setting. (Recall that a supplementary qualitative condition is necessary in this case to obtain a result.)

Proposition. Let $u: \Omega \rightarrow \mathbb{R}$ be of type 3 , with $\Omega \subseteq \mathbb{R}^{2}$ bounded. Then for $|\lambda| \gg 1$,

$$
\left|\int_{\left\{\frac{\partial^{2} u}{\partial x_{1} d x_{2}} \geq 1\right\}} e^{i \lambda u(x)} f_{1}\left(x_{1}\right) f_{2}\left(x_{2}\right) d x_{1} d x_{2}\right| \leq C \frac{(\log |\lambda|)^{\frac{1}{2}}}{|\lambda|^{\frac{1}{2}}}\left\|f_{1}\right\|_{2}\left\|f_{2}\right\|_{2}
$$

where $C$ depends on the diameter of $\Omega$ and $t_{3}(u)$.

The proof of this proposition is obtained by examining and reworking a similar result of Phong and Stein [PS1]. Hence we obtain:

Theorem 6. Let $\alpha$ be a multiindex in $\mathbb{Z}_{+}^{n}$ with at least two nonzero entries. Let $u: \Omega \rightarrow \mathbb{R}$ be of type $\max \{|\alpha|, 3\}$ with $\Omega \subseteq \mathbb{R}^{n}$ bounded. Then for $|\lambda| \gg 1$ and $\frac{1}{p_{i}}=1-\frac{\alpha_{i}}{|a|}$,

$$
\left|\int_{\left\{\left|D^{\alpha} u\right| \geq 1\right\}} e^{i \lambda u(x)} \prod_{i=1}^{n} f_{i}\left(x_{i}\right) d x_{i}\right| \leq C \frac{(\log |\lambda|)^{\frac{1}{2}}}{|\lambda|^{\frac{1}{|\alpha|}}} \prod_{i=1}^{n}\left\|f_{i}\right\|_{p_{i}, 1}
$$

and

$$
\int_{\left\{\left|D^{\alpha} u\right| \geq 1\right\}} e^{i \lambda u(x)} \prod_{i=1}^{n} f_{i}\left(x_{i}\right) d x_{i} \mid \leq C \frac{(\log |\lambda|)^{\frac{3}{2}}}{|\lambda|^{\frac{1}{|\alpha|}}} \prod_{i=1}^{n}\left\|f_{i}\right\|_{p_{i}}
$$

where $C$ depends on $\alpha, n, \Omega$ and $t_{\max \{|\alpha|, 3\}}(u)$.

Phong, Stein and Sturm in [PSS] have obtained a similar result to part (b) above, in the polynomial setting, with a logarithmic factor $(\log |\lambda|)^{n-\frac{1}{2}}$ instead of our $(\log |\lambda|)^{\frac{3}{2}}$. However, they have also shown that one may entirely remove the logarithmic factor in the above proposition at the expense of inserting a smooth cut-off function supported in $\left\{\frac{\partial^{2} u}{\partial x_{1} d x_{2}} \geq 1\right\}$, provided one is in the polynomial setting. (The constant will now depend also on the cut off and the total degree of $u$.) A corresponding improvement is then possible in Theorem 6 . We refer to $[\mathbf{C W}]$ for further details. 
4. Restricted-type estimates as in Theorems 5 and 6 , parts (a), are ideal if one has more data. Suppose $u: \Omega \rightarrow \mathbb{R}$ is of type $M$, and that $\alpha^{1}, \ldots, \alpha^{K}$ are multiindices with $\left|D^{\alpha^{j}} u\right| \geq \tau_{j}$ for each $1 \leq j \leq K$. What can we say about the multilinear operators? (We shall restrict ourselves here to the sublevel set operators, but similar remarks apply to the oscillatory integral operators.) By Theorem 5(a) we have

$$
\int_{\Omega \cap\{|u| \leq t\} \cap\left\{\left|D^{\alpha^{j}} u\right| \geq \tau_{j}\right\}} \prod_{i=1}^{n} \chi_{E_{i}}\left(x_{i}\right) d x_{i} \leq C\left(\frac{t}{\tau_{j}}\right)^{\frac{1}{\left|\alpha^{j}\right|}} \prod_{i=1}^{n}\left|E_{i}\right|^{1-\frac{\alpha_{i}^{j}}{\left|\alpha^{j}\right|}}
$$

for each $j=1, \ldots, K$. Suppose $\alpha=\sum_{j=1}^{K} \lambda_{j} \alpha^{j}$ is a convex combination of $\left\{\alpha^{1}, \ldots, \alpha^{K}\right\}$. Then by taking convex combinations of these estimates we obtain

$$
\int_{\Omega} \prod_{i=1}^{n} \chi_{E_{i}}\left(x_{i}\right) d x_{i} \leq C t^{\frac{1}{|\alpha|}} \prod_{i=1}^{n}\left|E_{j}\right|^{1-\frac{\alpha_{i}}{|\alpha|}} \prod_{j=1}^{K} \tau_{j}^{\frac{-\lambda_{j}}{|\alpha|}} .
$$

After a suitable interpolation argument whose details are in $[\mathbf{C W}]$ one obtains:

Theorem 7. Let $u: \Omega \rightarrow \mathbb{R}$ be of type $M$, and $\alpha^{1}, \ldots, \alpha^{K}$ multiindices with $\left|\alpha^{j}\right| \leq M$. If $\alpha$ is in the convex hull $\mathcal{A}$ of $\left\{\alpha^{1}, \ldots, \alpha^{K}\right\}$ but is not an extreme point of $\mathcal{A}$, then for $\frac{1}{p_{i}}=1-\frac{\alpha_{i}}{|\alpha|}$,

$$
\left|\int_{K \cap\{|u| \leq t\} \cap \bigcap_{j=1}^{K}\left\{\left|D^{\alpha^{j}} u\right| \geq 1\right\}} \prod_{i=1}^{n} f_{i}\left(x_{i}\right) d x_{i}\right| \leq C t^{\frac{1}{|\alpha|}} \prod_{i=1}^{n}\left\|f_{i}\right\|_{p_{i}}
$$

where $C$ depends on $n, \alpha, M$ and $t_{M}(u)$.

Thus, given $p_{i}$ with $\sum_{i=1}^{n} \frac{1}{p_{i}^{\prime}}=1$, the best decay rate $t^{\frac{1}{|\alpha|}}$ is obtained for the value of $|\alpha|$ where the line (in $\alpha$-space) $\alpha_{1} p_{1}^{\prime}=\cdots=\alpha_{n} p_{n}^{\prime}$ meets $\mathcal{A}$.

Similarly, if one wishes to obtain the best estimate (irrespective of the $p_{j}$ 's) of the form $t^{\delta}$, then $\delta^{-1}$ is given by the $\ell^{1}$-distance of 0 to the convex hull of $\left\{\alpha^{1}, \ldots, \alpha^{K}\right\}$, that is, $\delta^{-1}=\inf \{|\alpha|: \alpha \in A\}$. 


\section{References}

[CCW] A. Carbery, M. Christ and J. Wright, Multidimensional van der Corput and sublevel set estimates, J. Amer. Math. Soc. 12(4) (1999), 981-1015.

[CHS] A. Carbery, E. Hernández and F. Soria, Estimates for the Kakeya maximal operator on radial functions in $\mathbb{R}^{n}$, in: "Harmonic analysis" (Sendai, 1990), ICM-90 Satell. Conf. Proc., Springer, Tokyo, 1991, pp. 41-50.

[CW] A. Carbery and J. Wright, Multilinear sublevel set operators and multilinear interpolation, in preparation.

[F] C. Fefferman, Inequalities for strongly singular convolution operators, Acta. Math. 124 (1970), 9-36.

[G] I. R. GiBson, Ph.D. thesis in preparation, University of Edinburgh.

[PS1] D. H. Phong and E. M. Stein, On a stopping process for oscillatory integrals, J. Geom. Anal. 4(1) (1994), 105-120.

[PS2] D. H. Phong and E. M. Stein, The Newton polyhedron and oscillatory integral operators, Acta Math. 179(1) (1997), 105-152.

[PSS] D. H. Phong, E. M. Stein and J. A. Sturm, Multilinear level set operators, oscillatory integral operators and Newton polyhedra, Math. Ann. 319(3) (2001), 573-596.

[S] E. M. SteIn, "Harmonic analysis: real-variable methods, orthogonality, and oscillatory integrals", Princeton Mathematical Series 43, Monographs in Harmonic Analysis III, Princeton University Press, Princeton, NJ, 1993.

Department of Mathematics and Statistics

University of Edinburgh

James Clerk Maxwell Building

King's Buildings

Edinburgh EH9 3JZ

United Kingdom

E-mail address: carbery@maths.ed.ac.uk

E-mail address: wright@maths.ed.ac.uk 